\title{
A variant in the CASR gene (C.368T $>C$ ) causing hypocalcemia refractory to standard medical therapy
}

\author{
Diana Festas Silva1, Adriana De Sousa Lages ${ }^{(12,3}$, Joana Serra Caetano4, Rita Cardoso4, \\ Isabel Dinis ${ }^{4}$, Leonor Gomes ${ }^{1,2}$, Isabel Paiva ${ }^{1}$ and Alice Mirante ${ }^{4}$ \\ Endocrinology, Diabetes and Metabolism Department, Coimbra Hospital and University Centre, Coimbra, Portugal, \\ 2Faculty of Medicine of the University of Coimbra, Coimbra, Portugal, ${ }^{3}$ Endocrinology Department, Braga Hospital, \\ Braga, Portugal, and 4Pediatric Endocrinology, Diabetes and Growth Department, Coimbra Pediatric Hospital, \\ Coimbra, Portugal
}

Correspondence should be addressed to D Festas Silva Email

dianafsilva2@gmail.com

\section{Summary}

Hypoparathyroidism is characterized by low or inappropriately normal parathormone production, hypocalcemia and hyperphosphatemia. Autosomal dominant hypocalcemia (ADH) type 1 is one of the genetic etiologies of hypoparathyroidism caused by heterozygous activating mutations in the calcium-sensing receptor (CASR) gene. Current treatments for ADH type 1 include supplementation with calcium and active vitamin D. We report a case of hypoparathyroidism in an adolescent affected by syncope without prodrome. The genetic testing revealed a variant in the CASR gene. Due to standard therapy ineffectiveness, the patient was treated with recombinant human parathyroid hormone (1-34), magnesium aspartate and calcitriol. He remained asymptomatic and without neurological sequelae until adulthood. Early diagnosis and treatment are important to achieve clinical stability.

\section{Learning points:}

- Autosomal dominant hypocalcemia (ADH) type 1 is one of the genetic etiologies of hypoparathyroidism caused by heterozygous activating mutations in the calcium-sensing receptor (CASR) gene.

- The variant c.368T>C (p.Leu123Ser) in heterozygosity in the CASR gene is likely pathogenic and suggests the diagnosis of ADH type 1.

- Teriparatide (recombinant human parathyroid hormone 1-34) may be a valid treatment option to achieve clinical stability for those individuals whose condition is poorly controlled by current standard therapy.

\section{Background}

Hypoparathyroidism is a rare endocrine disorder characterized by low or inappropriately normal parathormone $(\mathrm{PTH})$ production, hypocalcemia and hyperphosphatemia $(1,2)$.

Up to $75 \%$ of cases arise from injury to the parathyroids during neck surgery.

The remaining $25 \%$ of cases are non-surgical or, even rarer, related to genetic disorders $(3,4)$.
Autosomal dominant hypocalcemia (ADH) type 1 (OMIM\# 601198) is one of the genetic etiologies of hypoparathyroidism and is caused by heterozygous activating mutations in the calcium-sensing receptor (CASR), located at chromosome 3q21.1, and represents the most common genetic form of isolated hypoparathyroidism $(5,6)$.

Activating CASR mutations cause a leftward shift in the calcium-PTH curve and consequently an increased CASR 
sensitivity to extracellular ionized calcium, which results in abnormal PTH suppression by normal extracellular calcium levels and hence hypocalcemia $(5,6)$.

$\mathrm{ADH}$ has a wide spectrum of clinical manifestations, ranging from asymptomatic forms to severe hypocalcemia-induced seizures. Most patients with $\mathrm{ADH}$ are asymptomatic or mildly symptomatic and, therefore, remain undiagnosed until adulthood, when hypocalcemia is incidentally detected $(2,7,8)$. Children may become symptomatic and present signs of neuromuscular irritability, especially during periods of stress and illness, which may be misdiagnosed as infection-induced events (8).

Complications of the disease are diverse, multisystemic and may include renal dysfunction due to nephrocalcinosis or lithiasis, ectopic calcifications of the basal ganglia, posterior subcapsular cataract, low bone turnover and increased bone density (1).

Typical biochemical features are mild to moderate hypocalcemia (normal range: 1.50-2.00 $\mathrm{mmol} / \mathrm{L}$ ), occasionally as low as $1.25 \mathrm{mmol} / \mathrm{L}$, hyperphosphatemia, hypomagnesemia, low or normal serum levels of PTH and normal to elevated levels of urinary calcium (8).

Current standard treatment includes supplementation with calcium and active vitamin D. Albumin-corrected serum calcium level should be kept as low as possible to relieve symptoms (5), with normalization of urinary calcium, and serum phosphorus should be maintained within the high-normal range, avoiding significant hypoor hypercalcemia $(3,9,10)$.

Although there are approved standard therapy, some individuals fail to meet treatment goals and require multiple hospitalizations for either treatment optimization or hypocalcemia-related acute or chronic complications (10).

Recombinant human parathyroid hormone (rhPTH 1-34) is a synthetic PTH approved for pharmacological control of osteoporosis but, so far, not for hypoparathyroidism. Teriparatide has been evaluated in adult and pediatric patients with postsurgical or permanent hypoparathyroidism from CASR activation mutations and appears to be safe and effective, despite scarce evidence of its benefits and safety for long-term use (1).

rhPTH (1-84) has been approved for hypoparathyroidism, excluding patients with ADH1 (5).

\section{Case presentation}

A 13-year-old male patient was admitted in the Emergency Department due to syncope during physical activity, without prodrome, that lasted less than $5 \mathrm{~min}$ and resulted in facial trauma. There was a gradual recovery of the state of consciousness, with amnesia for the event, but there was no reference to seizures, loss of sphincter control or other complaints.

He was born full-term via cesarean delivery, from nonconsanguineous Caucasian parents, with the following somatometric characteristics at birth - weight $4595 \mathrm{~g}$, length $53 \mathrm{~cm}$ and head circumference $40 \mathrm{~cm}$.

From his medical records, standard screening for metabolic diseases on the 7th day of life and family history were unremarkable.

Physical examination revealed a positive Chvostek sign; but no facial dysmorphisms, pathologic heart murmurs, subcutaneously calcifications or other mucocutaneous changes were observed. There was also no delay in psychomotor development.

\section{Investigation}

A basic analytical study revealed severe hypocalcemia 1.67 $\mathrm{mmol} / \mathrm{L}$ (NR: 2.19-2.66), low ionized calcium $0.80 \mathrm{mmol} / \mathrm{L}$ (NR: 1.2-1.38), hyperphosphatemia $3.06 \mathrm{mmol} / \mathrm{L}$ (NR: 0.95-1.75), hypomagnesemia $0.62 \mathrm{mmol} / \mathrm{L}$ (NR: 0.7-1.0) and low 25-Hydroxyvitamin D $12.3 \mathrm{ng} / \mathrm{mL}$ (NR: >30). Venous blood gas analysis did not unveil the presence of hypokalemic alkalosis.

Cardiac and renal ultrasounds were normal, as well as the ECG.

Cranial CT scan revealed multiple bilateral calcifications of the basal ganglia, particularly the pale nuclei and thalamus, which raised the hypothesis of metabolic disease (shown in Fig. 1). The EEG showed abnormal traces, suggesting brain dysfunction with some paroxysmal activity but with no signs of lateralization or focal abnormalities.

Ophthalmological and stomatological evaluations revealed posterior subcapsular cataract on the right side, as well as enamel hypoplasia (shown in Fig. 2).

\section{Treatment}

The patient was admitted to the pediatric ward for stabilization and started therapy with i.v. calcium gluconate $10 \% 0.5 \mathrm{mEq} / \mathrm{kg} / \mathrm{day}$, followed by calcium carbonate and calcitriol. He remained clinically asymptomatic and was discharged 16 days later on calcium carbonate $(3000 \mathrm{mg} /$ day) and calcitriol $(0.25 \mu \mathrm{g} /$ day) therapy.

Hewas referred to pediatric endocrinology consultation at a tertiary hospital for detailed etiological investigation with suspected diagnosis of hypoparathyroidism. 


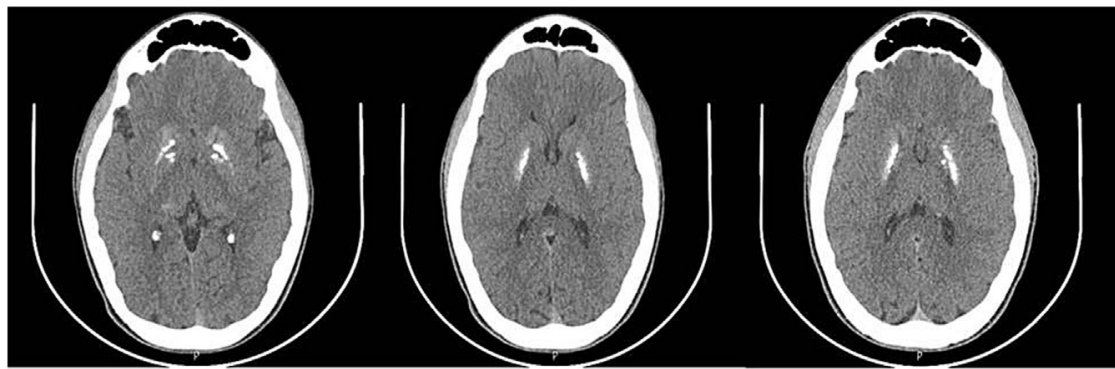

\section{Figure 1}

Basal ganglia and thalamus calcification. CT scan imaging showing multiple bilateral calcifications of the basal ganglia, in particular the pale nuclei, and thalamus.

At the first consultation, 7 days after discharge, the patient remained clinically asymptomatic, but further blood workup revealed hypocalcemia $1.57 \mathrm{mmol} / \mathrm{L}$ (NR: 2.19-2.66), hyperphosphatemia $2.94 \mathrm{mmol} / \mathrm{L}$ (NR: 0.95-1.75), hypomagnesemia $0.59 \mathrm{mmol} / \mathrm{L}$ (NR: 0.7-1.0), hypercalciuria $9.23 \mathrm{mmol} / 24 \mathrm{~h}$ (NR: 1.0-8.8), low 25-Hydroxyvitamin D $8.7 \mathrm{ng} / \mathrm{mL}$ (NR: $>30 \mathrm{ng} / \mathrm{mL}$ ) and inappropriately low PTH 4.0 pg/mL (NR: 16.0-87.0) (shown in Table 1). These biochemical abnormalities led to patient rehospitalization for therapeutic optimization.

During administration of i.v. calcium gluconate as an acute strategy for hypocalcemia correction, the patient developed significant calcification of the peripheral veins at the site of administration, which precipitated i.v. therapy suspension.

The doses of oral calcium, calcitriol and magnesium were gradually increased and sevelamer $800 \mathrm{mg}$ bid was added to control hyperphosphatemia.

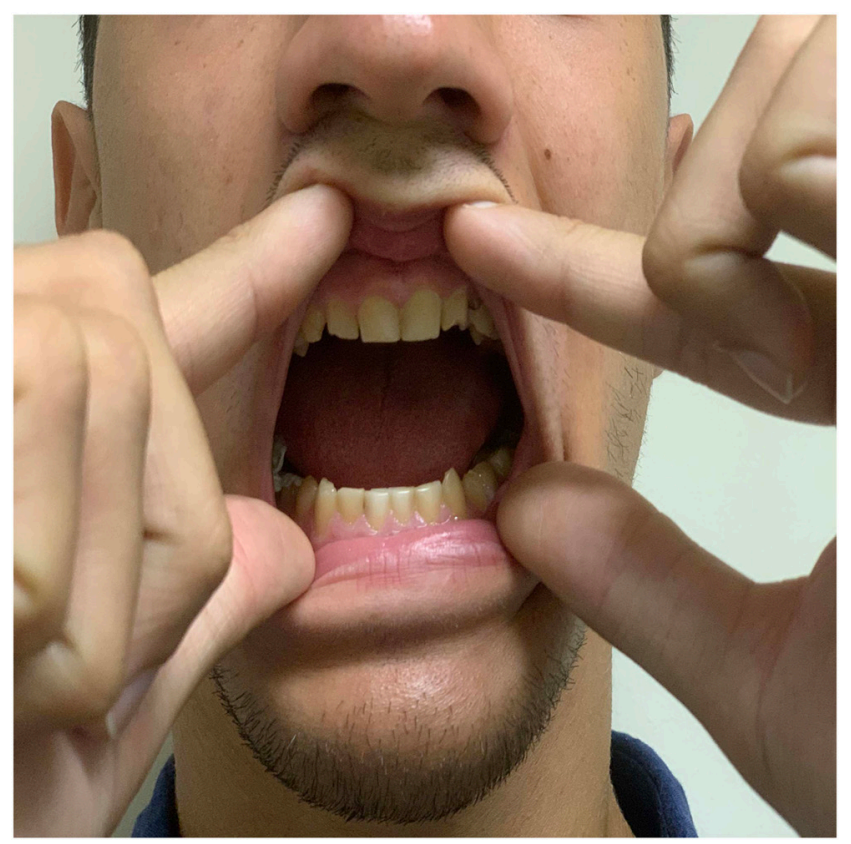

Figure 2

Enamel hypoplasia.
Despite the optimization and inward monitoring of adherence to oral therapy (calcium carbonate 9000 $\mathrm{mg}$ /day, sevelamer $2400 \mathrm{mg} /$ day, calcitriol $2 \mu \mathrm{g}$ /day and hydrochlorothiazide/amiloride $50-5 \mathrm{mg} /$ day), the patient hypocalcemia status was maintained $(1.60 \mathrm{mmol} / \mathrm{L}$ (NR: 2.19-2.66)), refractory to standard medical therapy.

As a last resource strategy for therapeutic optimization aiming to reduce long-term disease complications, the 14-year-old patient started on rhPTH (1-34) $20 \mu \mathrm{g}$ bid s.c., which was progressively increased to $20 \mu \mathrm{g}$ tid until the achievement of an adequate serum calcium level (2.02 mmol/L; NR: 2.19-2.66) (shown in Fig. 3). Upon the beginning of rhPTH (1-34) therapy, hydrochlorothiazide/ amiloride therapy was discontinued.

\section{Outcome and follow-up}

Since commencing rhPTH (1-34), the patient has remained asymptomatic and biochemically stable, his daily needs of oral calcium and calcitriol suffered a reduction of more than $50 \%$ and the sevelamer therapy was progressively suspended.

The pediatric patient maintained a regular follow-up in the Endocrinology Clinic until early adulthood with further adequate motor and cognitive development. There were no neurologic sequelae but additional renal ultrasound revealed enlarged kidneys with signs of diffuse medullary nephrocalcinosis (shown in Fig. 4).

The detection of nephrocalcinosis led to complementary patient re-education regarding the need to maintain a low-sodium diet and the importance of avoiding rhPTH therapy non-compliance.

During the transition from pediatric to adult care, additional investigations were performed to assess for autoimmune and genetic causes of hypocalcemia. Blood samples were collected first for anti-CASR antibodies detection, but after negative results, genetic analysis was performed for CASR, GNA11, GCM2 and PTH genes.

The study identified a variant, c.368T $>C$ (p.Leu123Ser) in heterozygosity in the CASR, which was predicted to be likely pathogenic by bioinformatics analysis. This 
Table 1 Initial investigation workup in the pediatric endocrinology at central hospital.

\section{Investigation}

Calcium (mmol/L)

Phosphorus (mmol/L)

Magnesium ( $\mathrm{mmol} / \mathrm{L})$

Sodium (mmol/L)

Potassium (mmol/L)

Parathormone $(\mathrm{pg} / \mathrm{mL})$

Calcium urine ( $\mathrm{mmol} / 24 \mathrm{~h})$

25-Hydroxyvitamin D (ng/mL)

Tubular phosphorus reabsorption rate

Creatinine $(\mathrm{mg} / \mathrm{dL})$

Estimated glomerular filtration rate $\left(\mathrm{mL} / \mathrm{min} / 1.73 \mathrm{~m}^{2}\right)$

ECG

Cardiac ultrasound

Renal ultrasound

variant has been described by Regala et al. in patients with autosomal dominant hypocalcemia and has not been identified in gnomAD population database. The variant affects a very conserved area of the CASR which codes its extracellular domain and where the majority of the variants that affect protein function are described. Along this line, and especially when associating this information with the data from the patient clinical condition, we considered this variant to be likely pathogenic.

\begin{tabular}{l}
\hline Result \\
\hline 1.57 \\
2.94 \\
0.59 \\
143.8 \\
4.3 \\
4.0 \\
9.23 \\
8.7 \\
$98 \%$ \\
0.58 \\
121.34 \\
No signs of hypocalcemia \\
No changes \\
No changes
\end{tabular}

\begin{tabular}{c}
\hline Normal range \\
\hline$(2.19-2.66)$ \\
$(0.95-1.75)$ \\
$(0.7-1.0)$ \\
$(135-145)$ \\
$(3.5-4.5)$ \\
$(16.0-87.0)$ \\
$(1.0-8.8)$ \\
$(>30)$
\end{tabular}

$(0.52-1.24)$

No family members reported symptoms associated with hypocalcemia or had a history of hypocalcemia detected during general practice appointments. The patient's mother refused to consent for genetic study and the patient's father had already died due to acute leukemia (at age 45 ).

The patient is currently 19-years old and is prescribed rhPTH (1-34) $20 \mu \mathrm{g}$ tid, magnesium aspartate $1229.6 \mathrm{mg} /$ day, calcitriol $0.25 \mu \mathrm{g} /$ day, low-sodium and low-

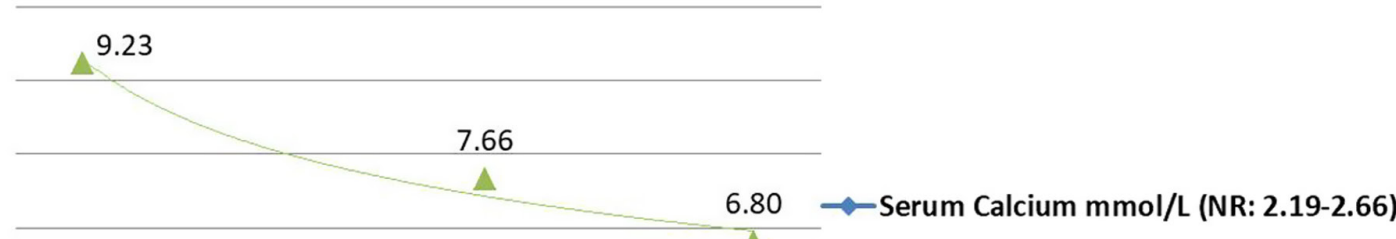

A

19-2.66)

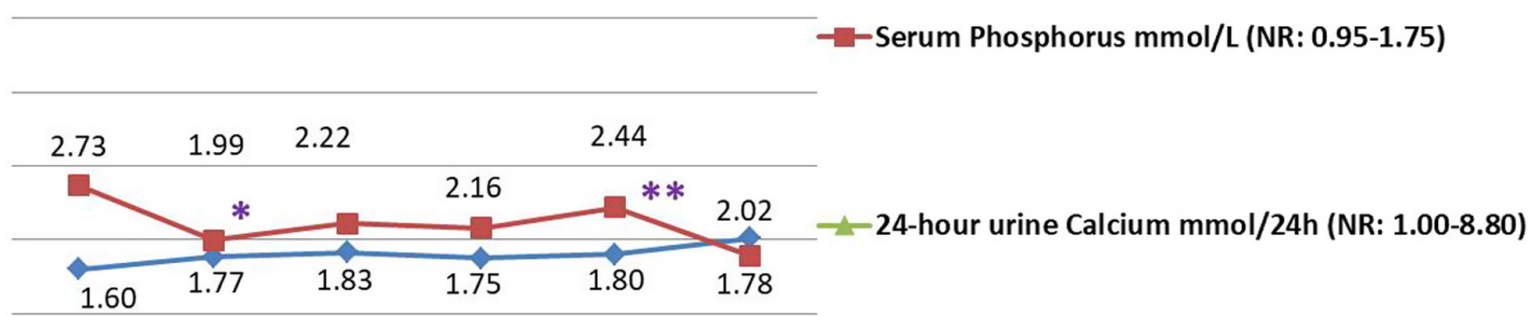

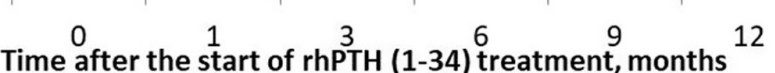

* rhPTH (1-34) $20 \mu \mathrm{g}$ bid; calcium carbonate $4500 \mathrm{mg} / \mathrm{day}$; calcitriol $1 \mu \mathrm{g} / \mathrm{day}$

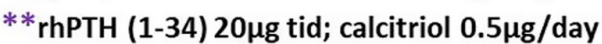

Figure 3

Variation in serum calcium, serum phosphorus and 24-h urine calcium levels after the start of rhPTH (1-34) treatment. Our patient's calcium and phosphorus levels approached the normal range after 12 months of treatment. 


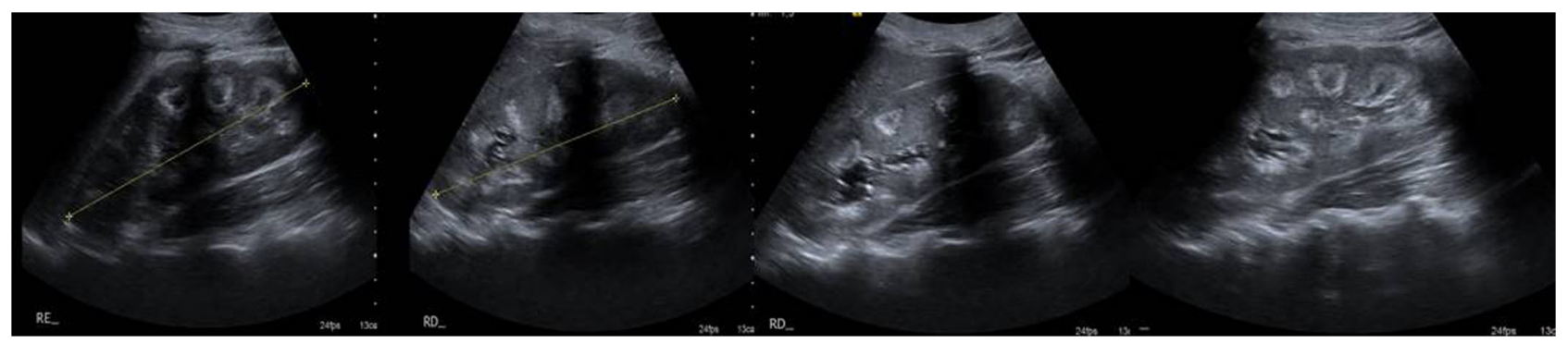

\section{Figure 4}

Renal medullary nephrocalcinosis. Kidneys with enlarged dimensions (about $13 \mathrm{~cm}$ of bipolar diameters), with signs of diffuse medullary nephrocalcinosis.

phosphorus diet and maintains regular endocrinology, ophthalmology and stomatology surveillance at a tertiary adult care center.

\section{Discussion}

The genetic analysis in the CASR gene confirmed the presence of a likely pathogenic variant, c.368T $>C$ (p.Leu123Ser) in heterozygosity suggesting the diagnosis of ADH type 1 . This genetic variant was not found in 50 normal controls withing the Portuguese population and was not present in the 1000 genomes database, indicating that it is not a common polymorphism and it may explain the patient's clinical manifestations (6).

In a work by Regala et al., published in 2015, this CASR gene variant was described in a boy with autosomal dominant hypocalcaemia, diagnosed at age 15 . Sequencing analysis in a sample from the mother suggested mosaicism for the same variant, although she was clinically and biochemically unaffected (6).

Some cases of de novo mutations and mosaicism in $\mathrm{ADH}$ have been identified in literature $(6,8)$ and may explain isolated cases with no familial history of hypocalcemia, as in this clinical case.

Due to the presence of hypocalcemia refractory to standard medical therapy and as a last resource strategy in therapeutic optimization and prevention of disease complications, the patient commenced therapy with rhPTH (1-34) at age 14.

Ever since, the patient has been clinically stable, maintaining mildly low serum calcium levels to minimize risks of complications.

No hospital admissions were required for management of hypocalcemia or any associated complications since the start of rhPTH (1-34) therapy, five years ago.

Although it is not currently approved for the treatment of hypoparathyroidism, some clinical trials have shown that recombinant $\mathrm{rhPTH}(1-34)$ can increase serum calcium while maintaining a normal level of urinary calcium excretion, holding promise as a treatment for $\mathrm{ADH}$ and refractory hypercalciuria (6). However, other studies have shown that PTH does not prevent the effects of the activated CASR in the kidney, which is reflected in abnormal urinary calcium excretion by patients during rhPTH (1-34) treatment (5).

Our patient, despite maintaining normal range urinary calcium levels during follow-up, developed renal diffuse medullary nephrocalcinosis as a complication, which was diagnosed at age 18 .

rhPTH (1-34) might be a potential treatment option for those individuals whose condition is not well controlled by the current standard therapy (with calcium, active vitamin $\mathrm{D}$, thiazide-type diuretics and/or magnesium as needed), in order to relieve their symptoms but also to minimize the risk of chronic hypocalcemia and hyperphosphatemia.

Declaration of interest

The authors declare that there is no conflict of interest that could be perceived as prejudicing the impartiality of the research reported.

\section{Funding}

This study did not receive any specific grant from any funding agency in the public, commercial or not-for-profit sector.

\section{Patient consent}

Written informed consent for publication of this report was obtained from the patient.

\section{Author contribution statement}

D F S carried out data collection and drafted the initial article. A S L investigated, followed up the treatment and critically reviewed the article. J S C, R C, I D, L G and I P critically reviewed the article. A M established clinical diagnosis, investigated and followed up the 
treatment and critically reviewed the article. All authors approved manuscript as submitted.

\section{Acknowledgements}

The authors acknowledge Dr Inês Castro, Dr Isa Salgado, Dr Jorge Basto and Dr Purificação Tavares for their important contribution regarding the genetic analysis.

\section{References}

1 Abate EG \& Clarke BL. Review of hypoparathyroidism. Frontiers in Endocrinology 20167 172. (https://doi.org/10.3389/fendo.2016.00172)

2 Sinnott BP. Hypoparathyroidism - review of the literature 2018. Journal of Rare Disorders: Diagnosis and Therapy 20184 1-7. (https://doi. org/10.21767/2380-7245.100180)

3 Clarke BL, Brown EM, Collins MT, Jüppner H, Lakatos P, Levine MA, Mannstadt MM, Bilezikian JP, Romanischen AF \& Thakker RV. Epidemiology and diagnosis of hypoparathyroidism. Journal of Clinical Endocrinology and Metabolism 2016101 2284-2299. (https://doi. org/10.1210/jc.2015-3908)

4 Bilezikian JP, Khan A, Potts JT, Brandi ML, Clarke BL, Shoback D, Juppner H, D’Amour P, Fox J, Rejnmark L, et al. Hypoparathyroidism in the adult: epidemiology, diagnosis, pathophysiology, target organ involvement, treatment, and challenges for future research. Journal of
Bone and Mineral Research 201126 2317-2337. (https://doi.org/10.1002/ jbmr.483)

5 Roszko KL, Bi RD \& Mannstadt M. Autosomal dominant hypocalcemia (hypoparathyroidism) types 1 and 2. Frontiers in Physiology 20167458. (https://doi.org/10.3389/fphys.2016.00458)

6 Regala J, Cavaco B, Domingues R, Limbert C \& Lopes L. Novel mutation in the CASR gene (p.Leu123Ser) in a case of autosomal dominant hypocalcemia. Journal of Pediatric Genetics 20154 29-33. (https://doi.org/10.1055/s-0035-1554979)

7 D'Souza-Li L. The calcium-sensing receptor and related diseases. Arquivos Brasileiros de Endocrinologia e Metabologia 200650 628-639. (https://doi.org/10.1590/s0004-27302006000400008)

8 Lienhardt A, Garabédian M, Bai M, Sinding C, Zhang Z, Lagarde JP, Boulesteix J, Rigaud M, Brown EM \& Kottler ML. A large homozygous or heterozygous in-frame deletion within the calcium-sensing receptor's carboxylterminal cytoplasmic tail that causes autosomal dominant hypocalcemia. Journal of Clinical Endocrinology and Metabolism 200085 1695-1702. (https://doi.org/10.1210/jcem.85.4.6570)

9 Shoback DM, Bilezikian JP, Costa AG, Dempster D, Dralle H, Khan AA, Peacock M, Raffael M, Silva BC, Thakker RV, et al. Presentation of hypoparathyroidism: etiologies and clinical features. Journal of Clinical Endocrinology and Metabolism 2016101 2300-2312. (https://doi. $\operatorname{org} / 10.1210 /$ jc.2015-3909)

10 Brandi ML, Bilezikian JP, Shoback D, Bouillon R, Clarke BL, Thakker RV, Khan AA \& Potts Jr JT. Management of hypoparathyroidism: summary statement and guidelines. Journal of Clinical Endocrinology and Metabolism 2016101 2273-2283. (https://doi.org/10.1210/jc.2015-3907)

Received in final form 2 September 2021

Accepted 10 November 2021 